\title{
Candida vaginitis among symptomatic pregnant women attending antenatal clinics in Mwanza, Tanzania
}

\author{
Martha F. Mushi ${ }^{*}$, Amani Mmole and Stephen E. Mshana
}

\begin{abstract}
Objective: This study was done to determine the patterns of Candida spp. causing vaginitis and associated factors among pregnant women attending antennal clinic in Mwanza, Tanzania.

Results: A total of 197 (65.6\%) out of 300 non-repetitive swabs had positive growth of Candida spp. Candida albicans 125 (63.4\%) was the most predominant isolated specie followed by C. tropicalis 35 (17.8\%) and C. glabrata 33 (16.8\%). Laboratory confirmed candida vaginitis was independently predicted by douching practices (OR 3.2, 95\% Cl 1.3-7.5 $P=0.007)$, history of antibiotics use (OR 1.8, 95\% Cl 1.02-3.0, $P=0.04)$ and low social economic status (OR 2.04, 95\% $\mathrm{Cl}$ 1.1-3.7 $\mathrm{P}=0.02$ ). About two-third of pregnant women with clinical features of vaginitis attending antenatal clinic in Mwanza, Tanzania were confirmed to have Candida vaginitis mainly caused by Candida albicans.
\end{abstract}

Keywords: Candida vaginitis, Candida albicans, Douching, Antibiotic use, Low social economic status

\section{Introduction}

Vulvo vaginal candidiasis or Candida vaginitis is the fungal infection of the female lower genital tract (vagina and vulva) caused by Candida spp. [1]. Candida vaginitis is the second most complain among women attending obstetrics and gynecological clinics worldwide [2, 3]. It is estimated that about $75 \%$ of women are affected with Candida vaginitis at least once during their life time with $15 \%$ of these cases present with a "cyclic recurrent type" which is defined as four or more episodes of Candida vaginitis in a year $[4,5]$.

Studies have shown that Candida albicans accounts for $80-95 \%$ of all episodes of candida vaginitis worldwide $[4,6]$. However, there is an increase of cases due to non-Candida albicans species led by Candida glabrata [7]. Other non-Candida albicans species reported to be associated with Candida vaginitis include: Candida tropicalis, Candida parapsilosis, Candida lusitaniae, Candida famata, Candida kefyr, Candida sake, Candida inconspicua, Candida valida, Candida colliculosa, Candida

\footnotetext{
*Correspondence: mushimartha@gmail.com

Department of Microbiology and Immunology, Catholic University

of Health and Allied Sciences, P. O. Box 1464, Mwanza, Tanzania
}

utilis, Candida catenulata, Candida lipolytica, Candida membranaefaciens, Candida intermedia and Candida globosa [7-9].

In East Africa, a study done in Aghakhan hospitalKenya reported $C$. albicans as the prominent species with prevalence of $69.3 \%$ followed by C. glabrata $12.9 \%$ [10]. In Tanzania, non-Candida albicans species were reported to contribute about $37 \%$ of Candida vaginitis cases [11]. However, data on azole susceptibility patterns and factors associated with Candida vaginitis are still limited. Here, we report the prevalence and factors associated with laboratory confirmed Candida vaginitis among pregnant women with symptoms of vaginitis attending antenatal clinics in Mwanza, Tanzania. Furthermore, data on the azole susceptibility patterns of these Candida spp. are reported.

\section{Main text}

This was a cross section study conducted from February to July 2016. The study was conducted at antenatal clinics of Nyamagana district hospital and Makongoro reproductive and child health clinic in Mwanza, Northwestern Tanzania. The selected clinics are representative 
of antenatal clinics that are highly populated in Mwanza serving more than 100 pregnant women per day.

Fungal isolation and fungal speciation by chromogenic agar were carried in the CUHAS microbiology laboratory and specie confirmation and antifungal susceptibility testing was done at Institute of Medical Microbiology, Gottingen, Germany.

All pregnant mothers attending antenatal clinics suspected of having Candida vaginitis and consented were recruited serially until the sample size was reached. For this study clinical Candida vaginitis was defined as having two or more of the following symptoms vaginal pruritus (itching), a thick odorless cottage cheese-like discharge and soreness [1]. The minimum sample size was obtained by the use of Kish Leslie formula [4].

\section{Culture for isolation and identifications of Candida spp.}

High vaginal swabs were cultured on Sabouraud's dextrose agar (SDA) supplemented with $50 \mu \mathrm{g} / \mathrm{ml}$ gentamicin and $50 \mu \mathrm{g} / \mathrm{ml}$ chloramphenicol (HiMediaMumbai, India) as previously described [12]. All yeasts isolated were identified to species level using chromogenic agar. Furthermore, 98 randomly picked isolates were confirmed by the use of matrix-assisted laser desorption ionization-time of flight (MALDI-TOF) mass spectrometry (Bruker Daltonics, Bremen, Germany) on extracted cells harvested from SDA as previous described [12, 13].

\section{In vitro susceptibility assays}

Antifungal susceptibility testing was done by establishing minimum inhibitory concentration (MIC) of fluconazole, voriconazole, posaconazole (Discovery Fine Chemicals, Bournemouth, United Kingdom), micafungin (Roth, Germany), caspofungin (Merck, US), and 5-fluorocytosine (Sigma Aldrich, US) following the guidelines laid down by the EUCAST [13].

\section{Data analysis and management}

All data collected were entered into Microsoft excel sheet for cleaning and coding then transferred to STATA version 13 for analysis according to the objectives of the study. Data were summarized into percentage for categorical variables while continuous variables (age and gestation age) were summarized as median with interquartile range (IQR). Logistic regression analysis was done to determine predictors of candida vaginitis. Statistical significant was considered when $P$ value was less than 0.05 with $95 \%$ confidence interval.

\section{Ethical considerations}

The protocol to conduct this study was approved by the joint Catholic University of Health and Allied Sciences/Bugando Medical Centre research ethics and review committee (CREC) with certificate number CREC/045/2014. Permission to conduct the study was sought from all hospital administrations. All patients were requested to sign the written informed consent before recruitment and patients' data were treated as confidential.

\section{Results}

A total of 300 pregnant women with mean age of $27 \pm 6.2$ years were recruited. The majority of women were married $275(91.7 \%)$, resided in urban areas 262 (87.3\%) and had primary school education 201 (67\%). About half of studied participants 168 (56\%) booked their first antenatal clinic on third trimester while 106 (35.3\%) and $26(8.7 \%)$ booked on second and first trimester, respectively. Their median gestation age at recruitment was 28 with interquartile range of 20-32 weeks. Most of the pregnant women had low social economic status 221 (74\%) as defined by having a fridge and television.

Laboratory confirmed Candida vaginitis was detected in $65.7 \%(197 / 300)$ of symptomatic pregnant women. Candida albicans was the most predominant detected Candida spp. 125 (63.4\%). A total of 72 (24\%) patients were diagnosed to have Candida vaginitis caused by nonCandida albicans spp. The predominant non Candida albicans spp. detected was Candida tropicalis 35 (17.8\%), Fig. 1. All isolated Candida albicans were highly susceptible to azole antifungal agents. However, Candida krusei were highly resistant to fluconazole and susceptible to other azole agents, Additional file 1.

\section{Factors associated with Candida vaginitis among pregnant women}

On univariable analysis, increase in gestation age OR 1.03, 95\% CI 1.01-1.06, $\mathrm{P}=0.04$, not having a secondary school education OR $2,95 \%$ CI $1.3-3.3, \mathrm{P}=0.008$, practicing douching OR $2.8,95 \%$ CI $1.28-6.25, \mathrm{P}=0.01$, history of antibiotic use in the past 2 weeks OR 1.8, 95\% CI 1.15-3.03, $\mathrm{P}=0.011$ and low social economic status OR $2.03,95 \%$ CI $1.21-3.42, \mathrm{P}=0.008$ were found to be associated with laboratory confirmed Candida vaginitis, Table 1.

On multivariable logistic regression analysis, having douching practices OR 3.2, 95\% CI 1.4-7.5, P $=0.007$, history of antibiotic use in the past 2 weeks OR 1.8, 95\% CI 1.02-3.0, $\mathrm{P}=0.02$ and low social economic status $\mathrm{OR}$ $2.04,95 \% \mathrm{CI} 1.1-3.7, \mathrm{P}=0.02$ were independent predictors of laboratory confirmed Candida vaginitis, Table 1.

\section{Discussion}

Candida vaginitis or thrush is the vaginal infection caused by yeast cells and commonly being reported among pregnant women of 20-40 years of age [2]. This has also been 


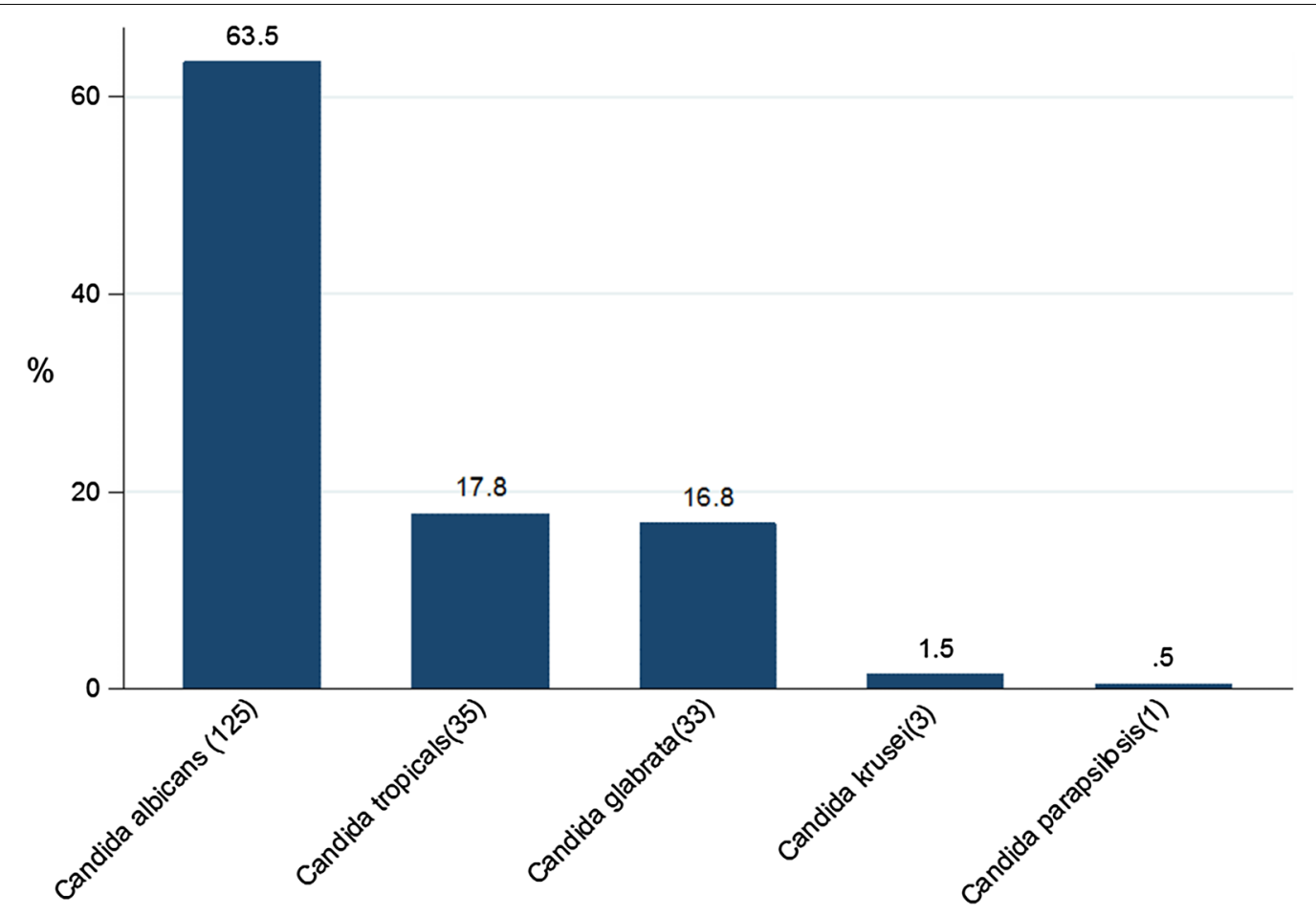

Fig. 1 Distributions of Candida spp. detected to cause Candida vaginitis

observed in the current study were by the median age of the pregnant women with clinical Candida vaginitis was 28 with interquartile range of 22-32 years. Physiological and tissue changes, due to reproductive hormones, which happen in young women especially during pregnancy, increase their susceptibility to Candida infection, in addition to adverse factors such as risky sexual behaviors. Previous study conducted in North America suggested that $70-75 \%$ of women can get at least one episode of Candida vaginitis in life time [6].

The current study established the prevalence of laboratory Candida vaginitis to be $65.7 \%$ among symptomatic pregnant women. This finding is similar to previous report from Nigeria which reported the prevalence of $62.2 \%$ [4] and slightly higher than $55.4 \%$ that was reported in Cameroon [14]. The different in study population could explain the differences. The study in Cameroon involved health non-pregnant women while the current study and the one from Nigeria involved pregnant women. The changes in sex hormones during pregnancy has been highly associated with the increases chances of Candida vaginitis [3].

As previous reported from different studies [2, 3, 11], the current study found $C$. albicans to be the most predominant specie causing Candida vaginitis. The virulence nature of Candida albicans in comparison to other Candida spp. could explain the findings [13]. Furthermore,
Candida albicans has been reported to be the most common Candida spp. colonizing the vaginal mucosa giving it high chance of causing infections in case of the presence of favorable conditions [10].

The use of antibiotic is known to suppress the bacterial normal flora and allow the overgrowth of yeast cells hence causing Candida vaginitis [15]. This was proven in the current study whereby the use of antibiotic was found to be an independent predictor of laboratory confirmed Candida vaginitis. Additionally in the current study douching practices which is also known to impair the growth of the vaginal microbiota [16-18] was found to independently predict the laboratory confirmed Candida vaginitis.

In the current study having low social economic status was also found to predict laboratory confirmed Candida vaginitis. Low social economic status is associated with poor hygiene [19]. Inability of the women to access basic needs include clean water and health care affect their hygienic practices. The poor hygienic practices can easily lead to vaginal candidiasis as previous observed in Cameroon [3].

About two-third of pregnant women with clinical features of vaginitis attending antenatal clinic in Mwanza, Tanzania were laboratory confirmed to have Candida vaginitis mainly caused by Candida albicans. Pregnant women with low social economic status (SES) with 
Table 1 Factors associated with vaginal Candida vaginitis among $\mathbf{3 0 0}$ studied pregnant women

\begin{tabular}{|c|c|c|c|c|c|c|}
\hline \multirow[t]{2}{*}{ Variable } & \multirow[t]{2}{*}{ Culture positive (\%) } & \multirow[t]{2}{*}{ Culture negative (\%) } & \multicolumn{2}{|l|}{ Univariate } & \multicolumn{2}{|l|}{ Multivariate } \\
\hline & & & OR $(95 \% \mathrm{Cl})$ & $P$ value & OR $(95 \% \mathrm{Cl})$ & $P$ value \\
\hline Age & 28 [22-32] & 26 [22-30] & $1.03(1.0-1.07)$ & 0.119 & $1.01(0.9-1.1)$ & 0.528 \\
\hline Gestation age & 28 [20-32] & 24 [20-32] & $1.03(1.01-1.06)$ & 0.041 & $1.03(0.9-1.1)$ & 0.068 \\
\hline \multicolumn{7}{|l|}{ Residence } \\
\hline Urban (262) & $167(63.7)$ & $95(36.3)$ & 1 & & & \\
\hline Rural (38) & $30(78.9)$ & $8(21.1)$ & $0.46(0.21-1.06)$ & 0.070 & - & - \\
\hline \multicolumn{7}{|l|}{ Marital status } \\
\hline Single (25) & $14(56.0)$ & $11(44.0)$ & 1 & & & \\
\hline Married (275) & $183(66.6)$ & $92(33.4)$ & $1.56(0.68-3.58)$ & 0.291 & - & - \\
\hline \multicolumn{7}{|l|}{ Education } \\
\hline Primary (201) & $141(70.1)$ & $60(29.9)$ & 1 & & & \\
\hline Secondary (89) & $48(53.9)$ & $41(46.1)$ & $2(1.3-3.3)$ & 0.008 & $1.25(0.8-2.5)$ & 0.411 \\
\hline College (10) & $8(80.0)$ & $2(20)$ & $1.7(0.4-8.3)$ & 0.5 .9 & $3.5(0.6-18.9)$ & 0.150 \\
\hline \multicolumn{7}{|l|}{ Occupation } \\
\hline Employed (184) & $114(67.0)$ & $70(38.0)$ & 1 & & & \\
\hline Unemployed (116) & $83(71.6)$ & $33(28.5)$ & $1.54(0.93-2.54)$ & 0.089 & - & - \\
\hline \multicolumn{7}{|l|}{ Yoghurt drinking } \\
\hline Yes (119) & $72(60.5)$ & $47(39.5)$ & 1 & & & \\
\hline No (181) & $125(69.1)$ & $56(30.9)$ & $1.46(0.89-2.36)$ & 0.128 & - & - \\
\hline \multicolumn{7}{|l|}{ Drinking alcohol } \\
\hline No (255) & $166(65.1)$ & 89 (34.9) & 1 & & & \\
\hline Yes (45) & $31(68.9)$ & $14(31.1)$ & $1.18(0.6-2.3)$ & 0.622 & - & - \\
\hline \multicolumn{7}{|l|}{ Gravidity } \\
\hline Prime gravid (73) & $43(58.9)$ & $30(41.1)$ & 1 & & & \\
\hline Gravid two (78) & $51(65.4)$ & $27(34.6)$ & $1.3(0.6-2.5)$ & 0.412 & - & - \\
\hline Multi gravid (149) & $103(69.1)$ & $46(38.9)$ & $1.56(0.9-2.8)$ & 0.133 & - & - \\
\hline \multicolumn{7}{|l|}{ Douching } \\
\hline No (28) & $12(42.9)$ & $16(57.1)$ & 1 & & & \\
\hline Yes (272) & $185(68.0)$ & $87(32.0)$ & $2.8(1.28-6.25)$ & 0.01 & $3.2(1.4-7.5)$ & 0.007 \\
\hline \multicolumn{7}{|l|}{ STI results* } \\
\hline Negative (233) & $159(68.2)$ & $74(31.8)$ & 1 & & & \\
\hline Positive (10) & $3(30)$ & $7(70)$ & $5.01(1.3-19.7)$ & 0.022 & $4.6(1.1-19.9)$ & 0.039 \\
\hline \multicolumn{7}{|l|}{ Antibiotic use } \\
\hline No (147) & $86(58.5)$ & $61(41.5)$ & 1 & & & \\
\hline Yes (153) & $111(72.6)$ & $42(27.4)$ & $1.8(1.15-3.032)$ & 0.011 & $1.8(1.02-3.0)$ & 0.020 \\
\hline \multicolumn{7}{|l|}{ HIV } \\
\hline Negative (290) & $189(65.2)$ & $101(34.8)$ & 1 & & & \\
\hline Positive (10) & $8(80.00)$ & $2(20.0)$ & $2.14(0.45-10.3)$ & 0.342 & - & - \\
\hline \multicolumn{7}{|l|}{ SES } \\
\hline High (82) & $44(53.7)$ & $38(46.3)$ & 1 & & & \\
\hline Low (218) & $153(70.2)$ & $65(29.8)$ & $2.03(1.21-3.42)$ & 0.008 & $2.04(1.1-3.7)$ & 0.02 \\
\hline
\end{tabular}

The box bracket [] is inter quartile range for gestation age while the curved brackets () is percentage

*Only 243 women tested for VDRL

SES Social economic status

history of antibiotic use and who are practicing douching are more likely to suffer Candida vaginitis. A large cohort study among the high risk groups is recommended to determine the effect of the vaginal candidiasis to the pregnancy. 


\section{Limitation}

The prevalence of laboratory Candida vaginitis in the current might been under estimated because the features used are not specific for Candida vaginitis.

\section{Supplementary information}

Supplementary information accompanies this paper at https://doi. org/10.1186/s13104-019-4793-z.

Additional file 1: Table S1. Antifungal susceptibility test data.

\section{Abbreviations}

BMC: Bugando Medical Centre; CUHAS: Catholic University of Health and Allied Sciences; EUCAST: European Committee of Antimicrobial Susceptibility Testing; OR: odd ratio; MALDI-TOF: Matrix-assisted Laser Desorption lonizationTime of Flight; SDA: Sabouraud's dextrose agar.

\section{Acknowledgements}

Authors would like to acknowledge the support provided by department of Microbiology and immunology of the Catholic University of Health and Allied Sciences, Mwanza, Tanzania.

\section{Authors' contributions}

MFM and SEM designed the work. AM \& MFM recruited patients, performed laboratory investigations and results interpretations. MFM and SEM analyzed and interpreted the data. MFM wrote the first draft of the manuscript which was critically reviewed by SEM. All authors read and approved the final manuscript.

\section{Funding}

None.

\section{Availability of data and materials}

The datasets used and/or analyzed during the current study available from the corresponding author on reasonable request.

\section{Ethics approval and consent to participate}

The protocol to conduct this study was approved by the joint CUHAS/BMC research ethics. All patients were requested to sign the written informed consent before recruitment was done. All patients' data were treated as confidential.

\section{Consent for publication}

None applicable.

\section{Competing interests}

The authors declare that they have no competing interests.

Received: 8 October 2019 Accepted: 11 November 2019 Published online: 27 November 2019

\section{References}

1. Sobel JD. Vulvovaginal candidosis. Lancet. 2007;369(9577):1961-71.
2. Foxman B, Muraglia R, Dietz J-P, Sobel JD, Wagner J. Prevalence of recurrent vulvovaginal candidiasis in 5 European countries and the United States: results from an internet panel survey. J Lower Genit Tract Dis. 2013;17(3):340-5.

3. Toua V, Djaouda M, Gaké B, Menye DE, Christie E, Tambe E, Akindoh W, Njiné T. Prevalence of vulvovaginal candidiasis amongst pregnant women in Maroua (Cameroon) and the sensitivity of Candida albicans to extracts of six locally used antifungal plants. Int Res J Microbiol. 2013;4(3):89-97.

4. Akah PA, Nnamani CE, Nnamani PO. Prevalence and treatment outcome of vulvovaginal candidiasis in pregnancy in a rural community in Enugu State, Nigeria. J Med Med Sci. 2010;1(10):447-52.

5. Sobel JD. Management of patients with recurrent vulvovaginal candidiasis. Drugs. 2003;63:1059-66.

6. Sobel J, Faro S, Force R. Vulvovaginal candidiasis: epidemiologic, diagnostic, and therapeutic considerations. Am J Obstet Gynecol. 1998;178:203-11.

7. Mohanty S, Xess I, Hasan F, Kapil A, Mittal S, Tolosa JE. Prevalence \& susceptibility to fluconazole of Candida species causing vulvovaginitis. Indian J Med Res. 2007;12:216-9.

8. Sobel JD. Genital candidiasis. Medicine. 2005;3(10):62-5.

9. Singh S. Treatment of vulvovaginal candidiasis. Clin Rev. 2003;136(9):26-30.

10. Mutua F, Revathi G, Machoki J. Species distribution and antifungal sensitivity patterns of vaginal yeasts. East Afr Med J. 2010;87(4):156-62.

11. Namkinga L, Matee M, Kivaisi K, Kullaya A, Mneney E. Identification of Candida strains isolated from Tanzanian pregnant women with vaginal candidiasis. East Afr Med J. 2005;82(5):226-34.

12. Mushi MF, Mtemisika Cl, Bader O, Bii C, Mirambo MM, Groß U, Mshana SE. High oral carriage of non-albicans Candida spp. among HIV-infected individuals. Int J Infect Dis. 2016;49:185-8.

13. Mushi MF, Bader O, Bii C, Groß U, Mshana SE. Virulence and susceptibility patterns of clinical Candida spp. isolates from a tertiary hospital, Tanzania. Med Mycol. 2018;57(5):566-72.

14. Kengne M, Shu SV, Nwobegahay JM, Achonduh O. Antifungals susceptibility pattern of Candida spp. isolated from female genital tract at the Yaoundé Bethesda Hospital in Cameroon. Pan Afr Med J. 2017;28(1):294

15. Mushi MF, Ngeta N, Mirambo MM, Mshana SE. Predictors of esophageal candidiasis among patients attending endoscopy unit in a tertiary hospital, Tanzania: a retrospective cross-sectional study. Afr Health Sci. 2018;18(1):66-71.

16. Heng LS, Yatsuya H, Morita S, Sakamoto J. Vaginal douching in Cambodian women: its prevalence and association with vaginal candidiasis. J Epidemiol. 2010;20(1):70-6.

17. Spence D. Candidiasis (vulvovaginal). BMJ Clin Evid. 2010;2010:0815

18. Amaral R, Giraldo PC, Gonçalves AK, Junior J-E, Santos-Pereira S, Linhares I, Passos MR. Evaluation of hygienic douching on the vaginal microflora of female sex workers. Int J STD AIDS. 2007;18(11):770-3.

19. Das P, Baker KK, Dutta A, Swain T, Sahoo S, Das BS, Panda B, Nayak A, Bara M, Bilung B. Menstrual hygiene practices, WASH access and the risk of urogenital infection in women from Odisha, India. PLoS ONE. 2015;10(6):e0130777.

\section{Publisher's Note}

Springer Nature remains neutral with regard to jurisdictional claims in published maps and institutional affiliations. 